\title{
Biomineralization of Nanoscale Single Crystal Hydroxyapatite
}

\author{
Tiffany Omokanwaye ${ }^{1}$, Otto C. Wilson, Jr. ${ }^{{ }^{*}}$ \\ ${ }^{1}$ Catholic University of America \\ BONE/CRAB Lab, Department of Biomedical Engineering, Washington, DC 20064
}

\author{
Ayelle Gugssa $^{2}$, Winston Anderson ${ }^{2}$ \\ ${ }^{2}$ Howard University, \\ Department of Biology, Washington, DC
}

* Corresponding Author -

(202)319-5822

wilsono@cua.edu 


\begin{abstract}
The chemical and physical characteristics of nanocrystalline hydroxyapatite particles which formed during the subcutaneous implantation of crab shell in Sprague-Dawley rats were studied using Selected Area Electron Diffraction (SAED) and High Resolution Transmission Electron Microscopy (HRTEM). The initial SAED characterization evidence indicated the presence of an amorphous calcium phosphate phase. The electron dense nanophase particles which formed in the wound healing zone displayed broad diffuse rings which usually indicate a low crystalline order or amorphous phase. High Resolution Transmission Electron Microscopy (HRTEM) revealed that these mineralized regions contained discrete single crystal particles less than $5 \mathrm{~nm}$ in size. Micrographs taken at successively higher magnifications revealed very small nanoparticles with a hexagonal arrangement of ion channels with characteristic spacing of 0.54 $\mathrm{nm}$ and $0.23 \mathrm{~nm}$. This study revealed that single crystal hydroxyapatite nanoparticles consisting of only a few unit cells formed via a biomineralization directed process.
\end{abstract}




\section{Introduction}

Hydroxyapatite (HAp) is the premier calcium phosphate biomineral that forms in mammalian hard tissues. The important role of HAp in various structural and metabolic processes has been studied extensively. However, many of the factors that contribute to the unique biological activity of HAp continue to remain a mystery. The important role of hydroxyapatite in biomedicine will continue to expand in scope as the fundamental biomineralization mechanisms which determine the size, morphology, surface and bulk chemistry, degree of crystalline order, and bioactivity of Hap are uncovered in more detail.

There have been many studies which have contributed greatly to our collective understanding of HAp biomineralization. Some of these findings have been translated into improved synthetic methods (Dorozhkin, 2013). In depth studies on the formation and structure of biological apatite have been limited due to its by its very small crystal size. Most of our present understanding is inferred from studies on synthetic materials where various influences such as ionic substitution effects can be studied in a systematic fashion (Legeros, 1965; Legeros et al., 1967; Fleet and Liu, 2007; Wang, Zuo, Huang, Hou, \& Li, 2010). A number of researchers have investigated calcium phosphate synthesized from precipitation reactions with high resolution transmission electron microscopy (HRTEM) and extremely small nanoHAp, with dimensions less than $10 \mathrm{~nm}$ have been reported (Rangavittal et al., 2000; Suvorova and Buffat, 2001; Li et al., 2007; Neira et al., 2009; Nudelman et al., 2010; Ospina et al., 2012). Biggemann et al. (2008) synthesized HAp with a wide size variation by precipitation and classified particles based on three size regimes with large particles (25 and $150 \mathrm{~nm}$ ) predominantly displayed, medium particles (approximately 5-20 nm), and a very limited population of small particles (less than $5 \mathrm{~nm}$ ). The smallest HAp crystals had a rectangular morphology which was approximately two times the HA unit cell 
parameters and represent the smallest synthetic HAp observed with HRTEM coupled with focalseries restoration. Smolen et al. (2013) used a combined microwave hydrothermal technique to synthesize HAp with an average size of $5.5 \mathrm{~nm}$ from calcium hydroxide and phosphoric acid.

This present study follows our previous work in evaluating the osteogenic potential of crab shell integument obtained from Calinectes Sapides (Beautiful Swimmer), the Blue Claw Crab (Wilson et al, 2012). The initial study was conducted by the subcutaneous implantation of

crushed crab shell in the abdominal region of Sprague-Dawley rats. Tissue samples were collected from the wound healing zone at various times points ranging from $1-48$ days. One of the most promising results from the study involved the observation of collagen bundles which were interspersed with an electron dense fine particulate phase that was hypothesized to be regions of mineralization. We provide high resolution electron microscopy evidence in this study to confirm the validity of this hypothesis based on an in depth analysis of 30 day implant samples. Furthermore, we establish that the mineral phase consists of nanosized single crystal hydroxyapatite particles with dimensions less than $5 \mathrm{~nm}$ and clearly visible lattice spots.

\section{Experimental Procedures}

\section{Materials}

Blue claw crab specimens were purchased from commercial vendors in MD and prepared for study as detailed below. Reagent $\mathrm{HCl}$ was obtained from Fisher Scientific (Pittsburgh, PA) and diluted with deionized water to prepare the demineralized crushed crab shell. Formaldehydeglutaraldehyde fixative (1:4 ratio) was used for tissue perfusion and fixation. Ethanol and Spurrs epoxy was used to embed mouse tissue samples for cutting of thin sections for optical and transmission electron microscopy (TEM) analysis using an ultramicrotome. 


\section{Crab Integument Preparation}

Carapace integument from fresh and fresh frozen Calinectes Sapides (Blue Claw Crab) specimens were removed and thoroughly cleaned with deionized water and allowed to dry. Carapace was initially crushed using a commercial coffee grinder using a pulse cycle. Subsequent size reduction was accomplished using a mortar and pestle. Crushed crab shell samples were washed and aged in absolute ethanol or $70 \mathrm{vol} \%$ ethanol as an initial step in sterilization. A portion of the crushed crab shell was demineralized by aging 2 grams of dry crab shell in $20 \mathrm{ml}$ of $0.5 \mathrm{M} \mathrm{HCl}$. The crab shell granules were allowed to react in the $\mathrm{HCl}$ for $1-3$ hours. The demineralized crab shell was washed with deionized water and finally rinsed with ethanol and allowed to dry in air.

\section{Characterization}

High Resolution Transmission Electron Microscopy (HRTEM) and nanodiffraction experiments were performed at the NISP characterization facility at the University of Maryland.

\section{In vivo crab shell implantation}

The procedures for crab shell implantation were reported in the initial study (Wilson et al, 2012). A subcutaneous incision was made in the abdominal region of anesthetized twenty eight day old Sprague-Dawley rats and approximately 10-20 mg of demineralized or untreated crab shell was implanted. The implant site was closed with stainless steel staples and rinsed with ethanol. After aging periods that ranged from a few days to eight weeks, the rats were euthanized and perfused with formaldehyde-glutaraldehyde fixative to fix the tissue. Tissue from the area of 
implantation was obtained and prepared for microscopy analysis by dehydration in successively higher ethanol/water solutions till a final dehydration step using $100 \%$ ethanol. The samples were then embedded in epoxy in preparation for ultramicrotoming. Approximately $90 \mathrm{~nm}$ thin sections were cut using a diamond knife and mounted on $\mathrm{C}$ coated $\mathrm{Cu}$ transmission electron microscopy (TEM) sample grids. Samples were stained with lead acetate and osmium tetroxide prior to imaging via TEM.

\section{Results and Discussion}

The wound healing response is a uniquely tailored cascade of orchestrated events. These events work in concert to coordinate tissue healing and remodeling. The response that followed the subcutaneous implantation of demineralized crab shell in the abdominal region of 28 day old Sprague-Dawley rats was assessed at different time periods. Our initial study (Wilson et al, 2012) highlighted short term events which occurred within the first week of implantation. The main cell interactions involved macarophage type cells in close proximity to a demineralized crushed Calinectes Sapides (Blue Claw) crab shell. Macrophages are considered to be the most important regulatory cell in the inflammatory reaction and become the predominant cell type during the latter part of the inflammatory phase. Macrophages are differentiated monocytes that work at sites of inflammation and infection by infiltrating the wound area and assisting in cleaning and removing damaged tissue debris and foreign particles. Macrophages possess numerous lysosomes for phagocytic function as they participate in the intracellular digestion of phagocytosed material. Activated macrophages also release several important growth factors, cytokines and chemotactic factors that attract fibroblasts to the wound area (Laguens and Gomez Dumm, 1969; Janeway et al., 2005; Li et al., 2007). 
The focus of this study is to investigate the identity and origin of the electron dense mineral phase which was found interspersed with collagen fibers and bundles. The implantation time spanned 28 days and fibroblast type cells are the predominant cell type present along with collagen fibers and bundles as shown in Figure 1. The fibroblasts display elongated cytoplasmic profiles and a prominent nucleus and are responsible for synthesizing specific types of collagen fibers. The collagen fibers display parallel and perpendicular (cross-section) orientations with respect to the plane of the paper.

A finely divided electron dense mineral phase is interspersed with the collagen fibers as shown in Figure 2. This electron dense mineral phase was also observed in extended clusters in regions adjacent to the collagen bundles as shown in Figure 3. The inset image of Figure 3 displays a selected area electron diffraction (SAED) pattern that was collected for the pictured region. The pattern displays characteristic broad and diffuse rings which indicate either fully amorphous structure or a low degree of crystalline order. This particular sample could be classified as possessing some structural order based on the presence of a few rings which were distinct enough to reasonably estimate the radius. A few $\mathrm{d}$ spacing values were calculated from ring radii using the equation $r d=\lambda L$ where $r$ represents the diffraction ring radius, $d$ is the interatomic plane spacing, $\lambda$ is the wavelength for electrons, and $\mathrm{L}$ is an instrumental parameter related to the distance between the sample and the camera. Values for $\mathrm{d}$ spacings of $0.22 \mathrm{~nm}$ and $0.18 \mathrm{~nm}$ were calculated from measured diffraction radii. The measured $\mathrm{d}$ spacing values are comparable with values for specific interatomic spacings for the family of planes with Miller Indices of (310) and (123) $\left(\mathrm{d}_{310}=0.2242 \mathrm{~nm}\right.$ and $\left.\mathrm{d}_{123}=0.1850 \mathrm{~nm}\right)$ for the carbonatehydroxyapatite phase obtained from the American Mineralogist Crystal Structure Database (Fleet, Liu, \& King, 2004). 
High resolution-transmission electron microscopy (HRTEM) was used to further characterize the mineral phase. The HRTEM image shown in Figure 4 shows some very interesting features. The mineral phase is composed of a large number of discrete particles that have dimensions less than $5 \mathrm{~nm}$. The particles have a seemingly equiaxed morphology at first glance. However, closer inspection reveals that the particles have extended boundaries which can be traced by focusing on the symmetric pattern of spots which appear in appropriately oriented particles. Some particles appear diamond-like in shape and lattice spots are visible for c-axis aligned particles. A characteristic spacing of $0.54 \mathrm{~nm}$ and $0.23 \mathrm{~nm}$ was measured between successive spots. This is in stark contrast to the SAED pattern which displayed diffuse rings (Figure 3) indicating that the particles are low crystalline order. The small size of the nanocrystals contributed to peak broadening and weakening of the scattered intensity. This led to our initial idea that the particles were amorphous or low crystalline order. However, the particle size was below the detection limit for SAED. The HRTEM studies confirmed the crystalline character of the nanoparticles.

A simplified model for the HAp unit cell is shown in Figure 5. It can be viewed as a single hexagon with three (3) phosphate, three (3) $\mathrm{Ca}(\mathrm{II})$, two (2) $\mathrm{Ca}(\mathrm{I})$ atoms (6 corner atoms each contributing $1 / 3$ of an atom) and a central hydroxyl molecule. The model can also be viewed as a central rhombohedral diamond shape with hydroxyl ions at the corners. The unit formula for this arrangement is $\mathrm{Ca}_{5}\left(\mathrm{PO}_{4}\right)_{3}(\mathrm{OH})$. The stoichiometry can be calculated based on geometric considerations where atoms or molecular ion groups that are completely within the bounds of the hexagon or diamond shape contribute one structural unit and those that fall on a boundary contribute a fractional structural unit. For the case of $\mathrm{Ca}_{5}\left(\mathrm{PO}_{4}\right)_{3}(\mathrm{OH})$, there are two (2) central $\mathrm{Ca}(\mathrm{I})$ atoms, one central $\mathrm{Ca}$ (II) atom and four (4) $\mathrm{Ca}(\mathrm{II})$ atoms on the boundary (each 
contributing $1 / 2$ of an atom) for a total of five (5) Ca atoms, one (1) central phosphate group and four (4) phosphates on the boundary (each boundary phosphate contributes $1 / 2$ of a phosphate group) for a total of three (3) phosphate molecules, and four corner hydroxyl groups (two (2) hydroxyl ions contributing $1 / 3$ and two (2) hydroxyl ions contributing $1 / 6$ ) for a total of one hydroxyl ion.

We have discovered much regarding the cellular and macromolecular processes which contribute to the rich symphony which plays during the wound healing process for hard tissue. However, there are still a vast array of additional phenomena and nuanced processes which direct our quest for deeper understanding of the bone healing and remodeling process. Even the seemingly simplest part of the bone healing paradox, the mineral phase, presents a wide number of challenging secrets which attract a great amount of research attention. The exact nature of the crystalline phase that forms during the formation, healing, and remodeling of bone has been the subject of much study over the past 200 years (Dorozhkin, 2013). One of the key points in defining bone and its unique structural and functional aspects is that advances in characterization techniques lead to refinements in our understanding of fundamental characteristics and potential mechanisms. Amorphous or non-crystalline solids possess short range or local order (Termine \& Posner, 1967). Suvorova and Buffat (2001) demonstrated that the XRD method has limitations in the phase analysis of small particles. Even selected area electron diffraction patterns taken from relatively large samples can be interpreted erroneously due to size effects.

One of the most fundamental questions involves the crystalline nature of calcium phosphate based biominerals. There have been many theories regarding biomineralized calcium phosphate phases since the early work of key pioneers. Robinson and Watson (1955) suggested that a considerable portion of newly formed mineral in young bone was not crystalline but an 
amorphous-like precipitate. Posner and collaborators (Termine and Posner, 1967; Eanes et al., 1965) used the data obtained from x-ray diffraction and electron microscopy to point to the fact that bone mineral is a mixture of amorphous calcium phosphate (ACP) and crystalline apatite. Posner and Betts (Betts and Posner, 1974; Betts et al., 1975; Posner and Betts, 1975) later proposed a model structure for ACP which involved a $\mathrm{Ca} 9\left(\mathrm{PO}_{4}\right)_{6}$ cluster known as Posner's cluster, that is present in crystalline HAp. Historic models of HAp formation consider that biogenic minerals have a precursor phase that can be amorphous or composed of a different mineral phase. In the case of bone apatite, it has been found that minute round particles (approximately 1-2 $\mathrm{nm}$ in diameter and similar to Posner's Clusters) are present in conjugation with type I collagen molecule and these clusters can be associated with early HAp nanocrystal formation.

Hydroxyapatite typically forms needle-like or lathe-like crystals. Posner's clusters are roughly spherical with a diameter of $1 \mathrm{~nm}$ and it may be speculated that they play a role as a transient precursor and possible building block for ACP formation instead of undergoing complete ionic solvation during ACP to HAp transformation (Yin \& Stott, 2003). Our experiments at this time don't allow us to know whether the single crystal HAp nanoparticles that formed during this study went through an amorphous to crystalline transformation. However, the present results demonstrate that higher resolution electron microscopes of today allow us to visualize smaller scale atomic level features in more detail. This important tool will help us to further understand the biomineralization phenomena related to the mechanisms for controlling the nucleation and size control of Hap over multiple size scales. 


\section{Conclusions}

Nanocrystalline particles with characteristics similar to hydroxyapatite formed in the wound healing region where crushed crab shell was subcutaneously implanted in rats. The particles appeared amorphous via SAED. However, HRTEM imaging revealed that the fine particles which were less than $5 \mathrm{~nm}$ in size were actually single crystal particles which exhibited spots that were arranged in a hexagonal array for c-axis oriented particles. The spot distance between spots in a diamond shaped geometrical array was approximately $0.54 \mathrm{~nm}$ for the major axis and $0.23 \mathrm{~nm}$ for the minor axis. These dimensions correspond very closely with expected spacing for atoms in hydroxyapatite crystal lattice.

\section{Acknowledgements}

The authors acknowledge the support of the National Science Foundation (O.C. Wilson, Jr., Career Award DMR-0645675). SEM analysis was performed at the NISP Microanalysis facility at UMCP.

\section{References}

Betts F, Blumenthal NC, Posner AS, Becker GL, and Lehninger AL. (1975). Atomic structure of intracellular amorphous calcium phosphate deposits. Procceedings of the National Acadeny of Sciences, 72(6):2088-2090.

Betts F and Posner AS. (1974). An X-ray radial distribution study of amorphous calcium phosphate. Mater. Res. Bull., 9:353-360. 
Biggemann D, Prado da Silva MH, Rossi AM, and Ramirez AJ. (2008). High-Resolution Transmission Electron Microscopy Study of Nanostructured Hydroxyapatite. Microscopy and Microanalysis, 14:433-438.

Dorozhkin, SV. (2013). Nanodimensional and nanocrystalline calcium orthophosphates International Journal of Chemistry and Material Science. 1(6):105-174.

Dorozhkin, SV. (2013). A detailed history of calcium orthophosphates from 1770s till 1950. Materials Science and Engineering C. 33(6):3085-3110

Eanes ED, Gillessen IH, and Posner AS. (1965). Intermediate states in the precipitation of hydroxyapatite. Nature, 208:365-367.

Fleet ME, Liu X, and King PL. (2004). Carbonate-hydroxylapatite. American Mineralogist, 89:1422-1432.

Fleet ME and Liu X. (2007). Coupled substitution of type A and B carbonate in sodiumbearing apatite. Biomaterials, 28:916-926.

Janeway CH, Travers P, Walport M, and Shomchik MJ. (2005). Immuno Biology: the immune system in health and disease (6th Edition ed.). New York: Garland Science.

Laguens, RP and Gomez Dumm CL. (1969). Atlas of Human Electron Microscopy. Saint Louis: The C.V. Mosby Company.

LeGeros RZ. (1965). Effect of carbonate on the lattice parameters of hydroxyapatite. Nature, 4982:403-404.

LeGeros RZ, Trautz OR, LeGeros JP, Klein E, and Schirra WP. (1967). Apatite Crystallites: Effect of Carbonate on Science, 155:1409-1411.

Li J, Chen J, and Kirsner R. (2007). Pathophysiology of acute wound healing. Clinics in Dermatology, 25:9-18. 
Li ZY, Lam WM, Yang C, Xu B, Ni GX, Abbah SA, Cheung KMC, Luk KDK, Lu WW. (2007). Chemical composition, crystal size and lattice structural changes after incorporation of strontium into biomimetic apatite. Biomaterials, 28:1452-1460.

Neira IS, Kolen'ko YV, Lebedev OI, Van Tendeloo G, Gupta HS, Matsushita N, Yoshimura M and Guitián F. (2009). Rational synthesis of a nanocrystalline calcium phosphate cement exhibiting rapid conversion to hydroxyapatite. Materials Science and Engineering $C$ vol. 29(7):2124-2132.

Nudelman F, Pieterse K, George A, Bomans PHH, Friedrich H, Brylka LJ, Hilbers PAJ, de With G and Sommerdijk NAJM. (2010). The role of collagen in bone apatite formation in the presence of hydroxyapatite nucleation inhibitors. Nature Materials, 9:1004-1009.

Ospina CA, Ramirez AJ, Farina M, Ellis, DE, and Rossi, AM. (2012). Experimental evidence and structural modeling of nonstoichiometric $\left(\begin{array}{lll}0 & 1 & 0\end{array}\right)$ surfaces coexisting in hydroxyapatite nano-crystals. Colloids and Surfaces B: Biointerfaces, 89:15-12.

Posner AS. and Betts F. (1975). Synthetic amorphous calcium phosphate and its relation to bone mineral structure. Accounts of Chemical Research, 8:273-281.

Rangavittal N, Landa-Canovas AR, Gonzalez-Calbet, JM, and Vallet-Reg1 M. (2000). Structural study and stability of hydroxyapatite and $\beta$-tricalcium phosphate: Two important bioceramics. Journal of Biomedical Materials Research, 51:660-668.

Robinson RA and Watson ML. (1955). Crystal-collagen relationships in bone as observed in the electron microscope: III. Crystals and collagen morphology. Annals of the New York Academy of Sciences, 60:596-628.

Smolen D, Chudoba T, Malka I, Kedzierska A, Lojkowski W, Swieszkowski W, Kurzydlowski KJ, Kolodziejczyk-Mierzynska M, and Lewandowska-Szumiel M. (2013). Highly 
biocompatible, nanocrystalline hydroxyapatite synthesized in a solvothermal process driven by high energy density microwave radiation. Inter. J. Nanomedicine, 8:653-668.

Suvorova, EI and Buffat, PA. (2001). Electron diffraction and high resolution transmission electron microscopy in the characterization of calcium phosphate precipitation from aqueous solutions under biomineralization conditions. Eur. Cells Mater. 1:27-42.

Termine JD and Posner AS. (1967). Amorphous/Crystalline interrelationships in bone mineral. Calcified Tissue Research, 1:8-23.

Wang XY, Zuo Y, Huang D, Hou XD, and Li YB. (2010). Comparative study on inorganic composition and crystallographic properties of cortical and cancellous Bone. Biomedical and Environmental Sciences, 23:473-480.

Wilson, Jr. OC, Gugssa A, Mehl P, and Anderson W. (2012). An initial assessment of the biocompatibility of crab shell for bone tissue engineering, Mater. Sci. Eng. C 32:78-82.

Yin X and Stott MJ. (2003). Biological calcium phosphates and Posner's clusters. Journal of Chemical Physics, 118(8):3717-3723. 


\section{Figure Captions}

Figure 1. Transmission electron microscopy image of tissue sample obtained from in vivo study of demineralized crushed Calinectes Sapides (Blue Claw) crab shell implanted subcutaneously in abdominal region of 28 day old Sprague-Dawley rats (28 day implantation time). Collagen fibers, collagen bundles, and fibroblasts are shown in the image.

Figure 2. Transmission electron microscopy image of collagen bundles that formed after subcutaneous implantation of mineralized crab shell for 28 days. Note the collagen fibers with the characteristic $67 \mathrm{~nm}$ banding pattern and the appearance of electron dense mineral phase interspersed among collagen bundles and in adjacent regions.

Figure 3. Transmission electron microscopy image of clear region containing electron dense mineral phase with an inset selected area electron diffraction (SAED) image depicting a representative amorphous or low crystalline order pattern.

Figure 4. High Resolution Transmission Electron Microscopy image of the nanocrystalline mineral phase. Particle dimensions are less than $5 \mathrm{~nm}$ and spots which display hexagonal symmetry are indicative of columns of atoms in the crystal lattice.

Figure 5. Simplified two dimensional (2-D) Model for the hydroxyapatite (HAp) unit cell that shows hexagonal spot array that depicts relative positions for $\mathrm{Ca}(\mathrm{I}), \mathrm{Ca}(\mathrm{II})$, phosphate, and hydroxyl ion channels. 


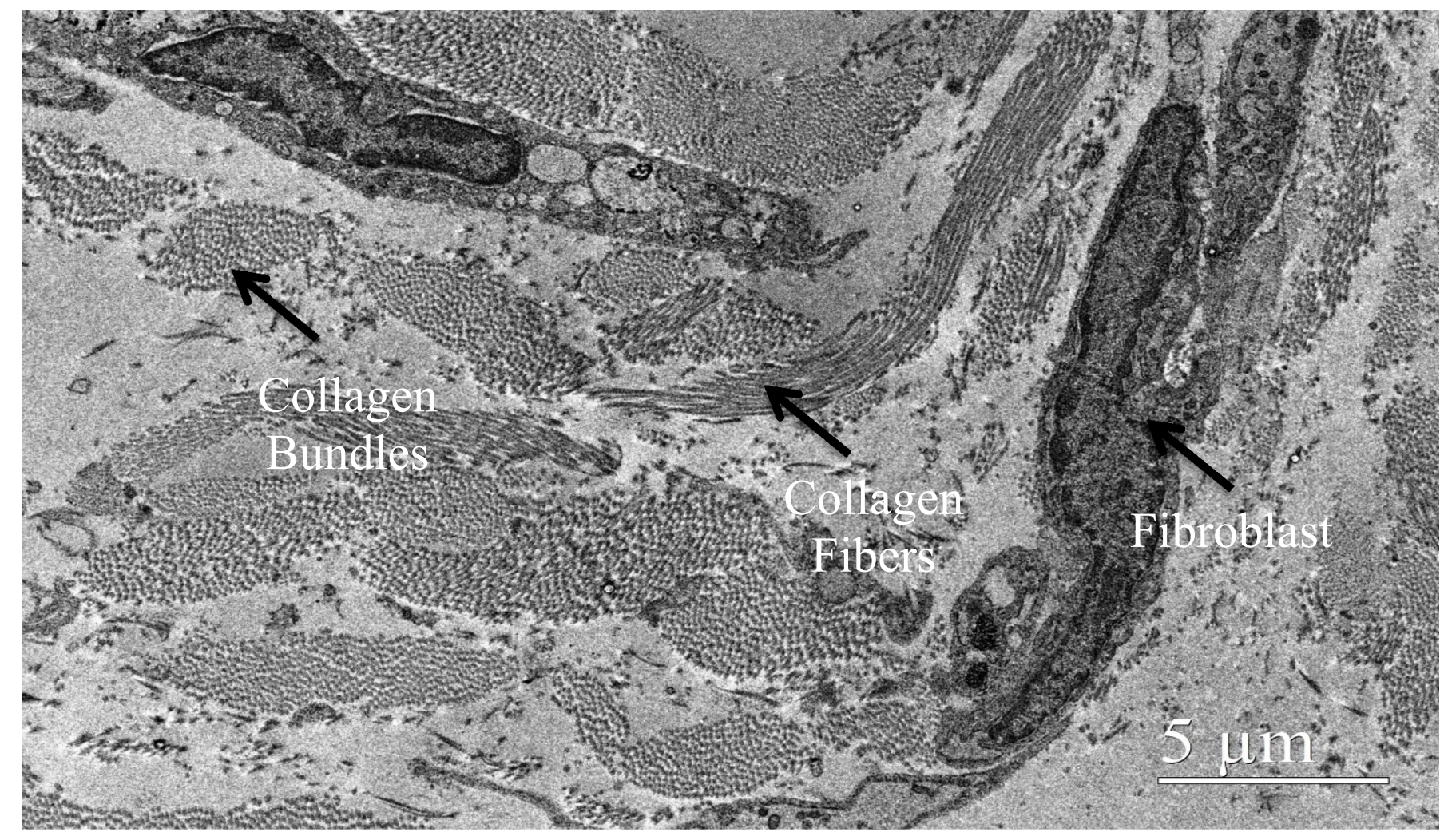

Figure 1 Transmission electron microscopy image of tissue sample obtained from in vivo study of demineralized crushed Calinectes Sapides (Blue Claw) crab shell implanted subcutaneously in abdominal region of 28 day old Sprague-Dawley rats (28 day implantation time). Collagen fibers, collagen bundles, and fibroblasts are shown in the image. 


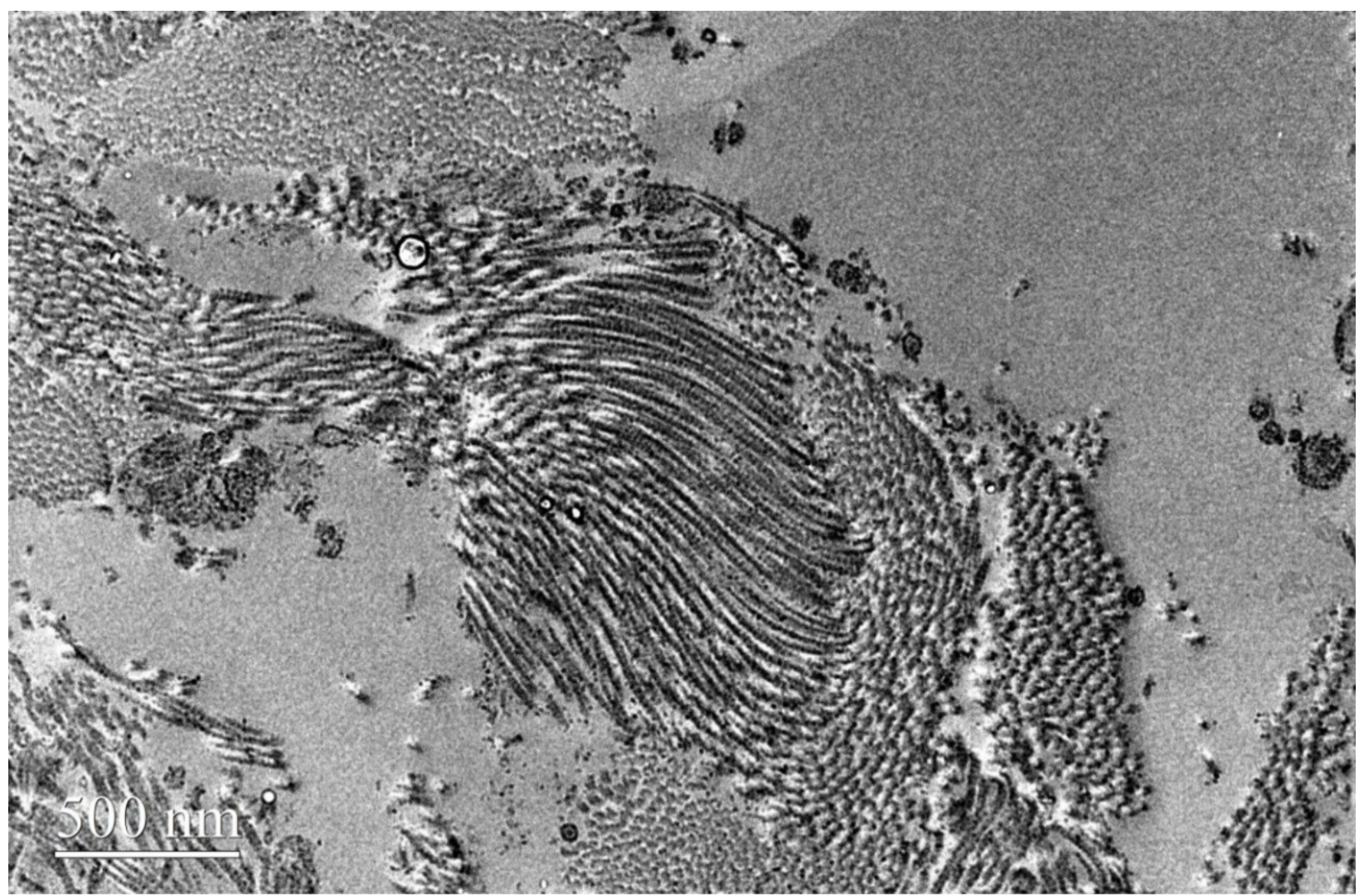

Figure 2. Transmission electron microscopy image of collagen bundles that formed after subcutaneous implantation of mineralized crab shell for 28 days. Note the collagen fibers with the appearance of electron dense mineral phase interspersed among collagen bundles and in adjacent regions. 


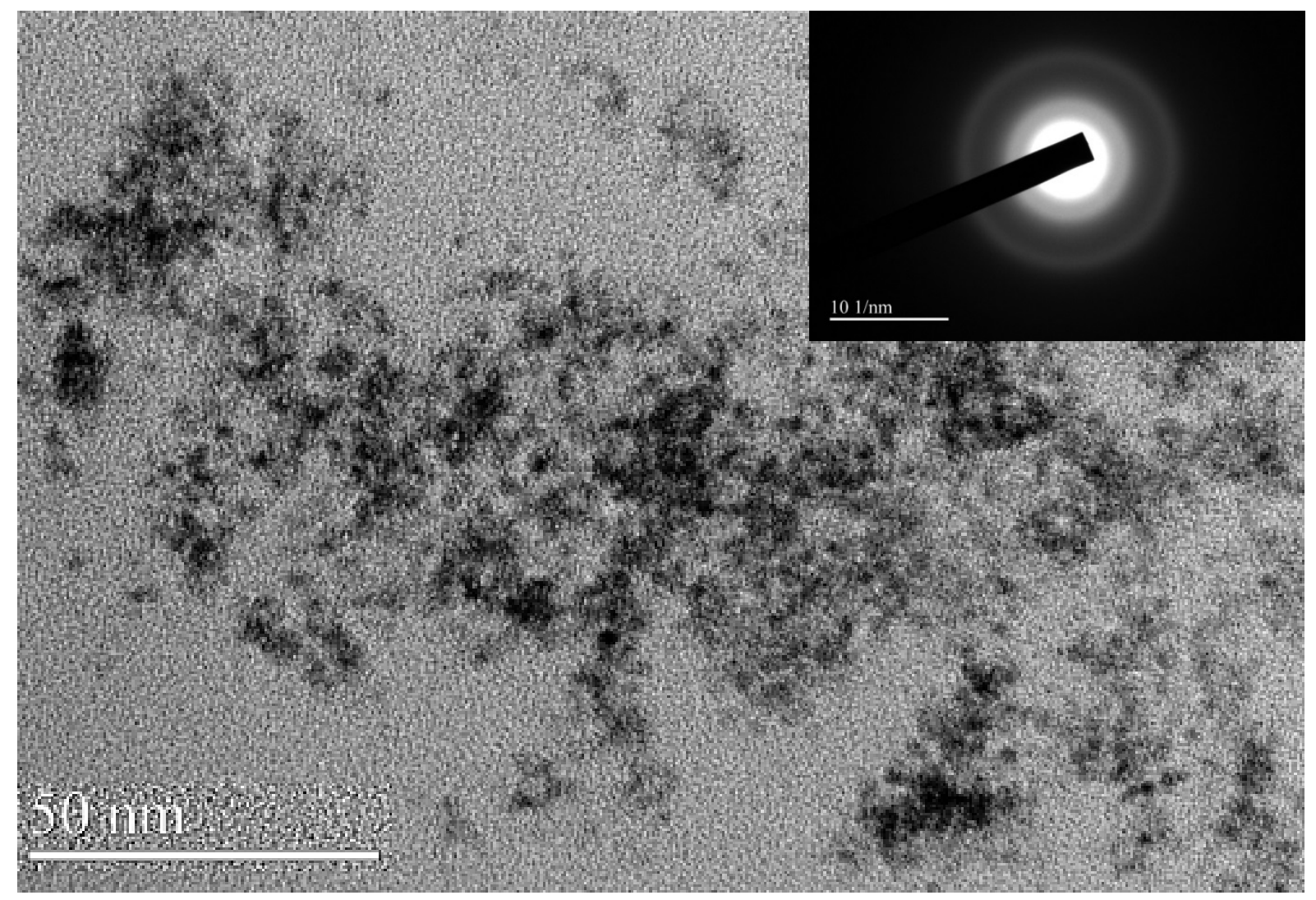

Figure 3. Transmission electron microscopy image of clear region containing electron dense mineral phase with an inset selected area electron diffraction (SAED) image depicting a representative amorphous or low crystalline order pattern. 


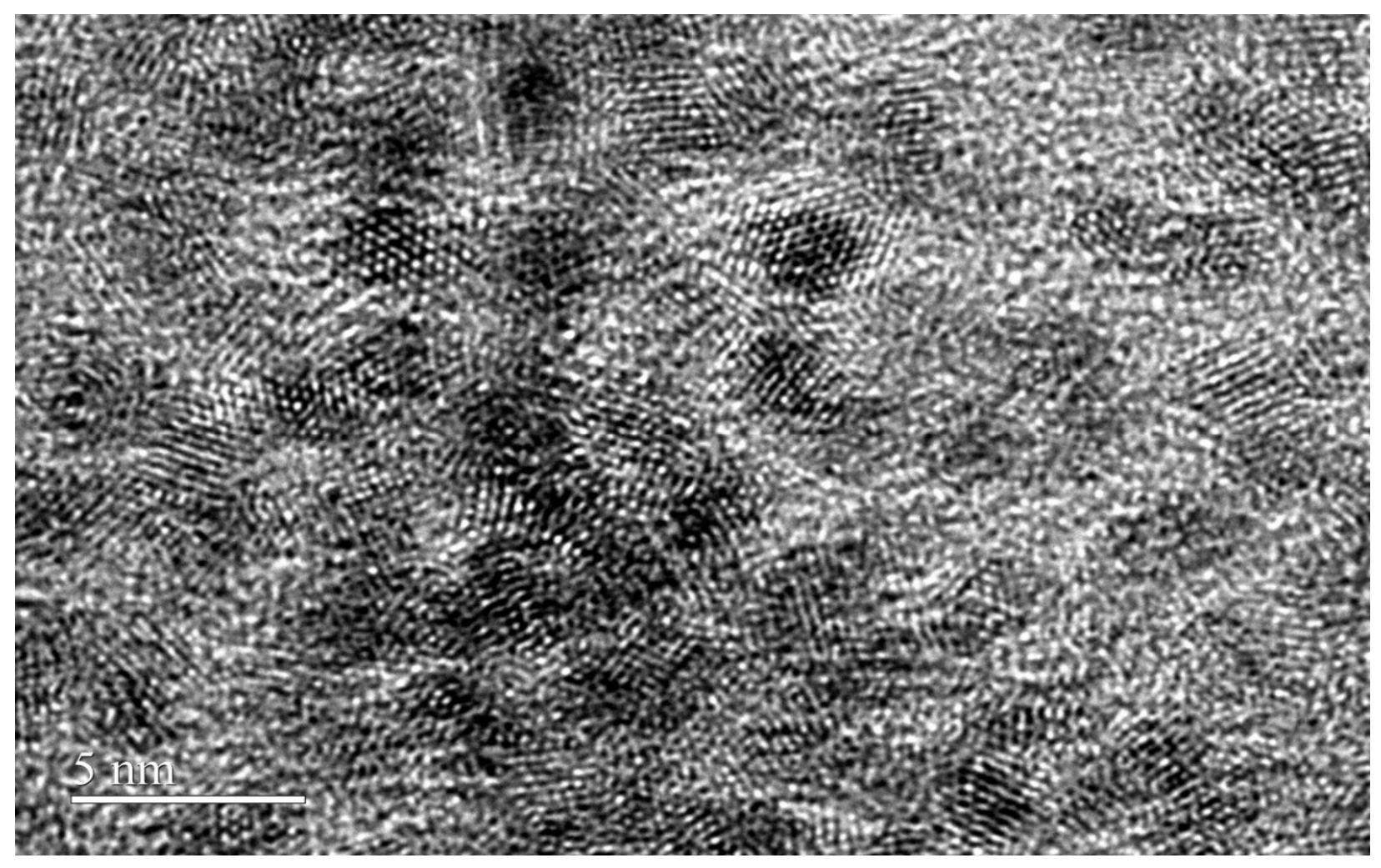

Figure 4. High Resolution Transmission Electron Microscopy image of the nanocrystalline mineral phase. Particle dimensions are less than $5 \mathrm{~nm}$ and spots which display hexagonal symmetry are indicative of columns of atoms in the crystal lattice. 


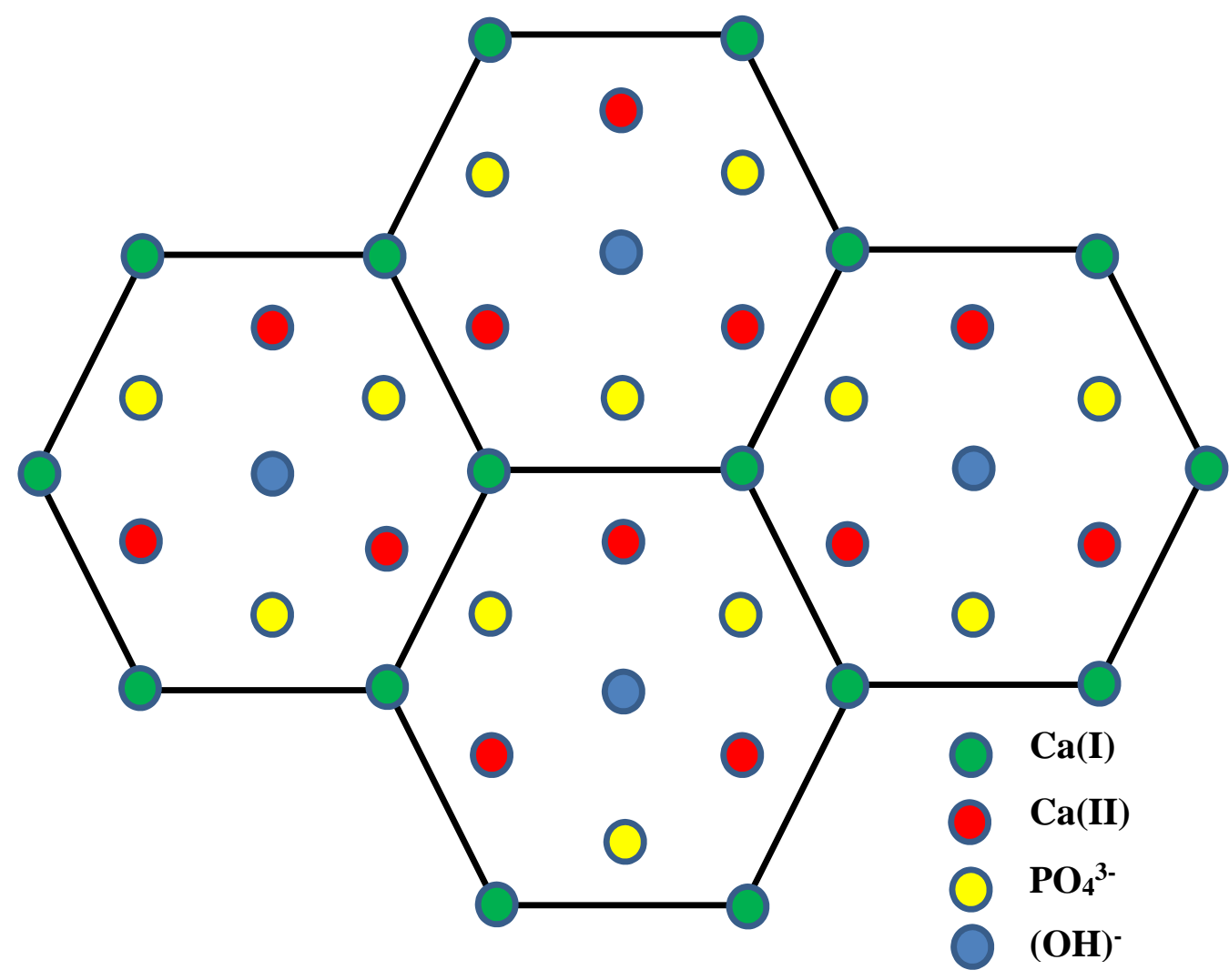

Figure 5. Simplified two dimensional (2-D) Model for the hydroxyapatite (HAp) unit cell that shows hexagonal spot array that depicts relative positions for $\mathrm{Ca}(\mathrm{I}), \mathrm{Ca}(\mathrm{II})$, phosphate, and hydroxyl ion channels. 